\title{
Transcriptional regulatory networks of methanol-independent protein expression in Pichia pastoris under the AOX1 promoter with trans-acting elements engineering
}

\author{
Lei Shi ${ }^{1}$, Jinjia Wang ${ }^{1}$, Xiaolong Wang ${ }^{1}$, Yuanxing Zhang ${ }^{1,2}$, Zhiwei Song ${ }^{3,4}$, Menghao Cai ${ }^{1}$
} and Xiangshan Zhou ${ }^{1 *}$

\begin{abstract}
To explore the differences in the intracellular transcriptional mechanism in carbon-derepressed and wild-type Pichia pastoris strains fed with three different carbon sources. RNA in carbon-derepressed ( $\Delta$ mig1 $\triangle$ mig2 $\Delta \mathrm{nrg} 1-M i t$; Mut) and wild-type (WT) P. pastoris fed with three different carbon sources (dextrose, glycerol, and methanol) were sequenced. Differentially expressed genes (DEGs) associated with these carbon sources were obtained and clustered into modules using weighted gene co-expression network analysis (WGCNA). Signaling pathway enrichment analysis was performed using KEGG, and protein to protein interaction (PPI) network was also constructed. A total of 2536 DEGs were obtained from three intersections, and some of them were enriched in carbon sources and involved in carbon metabolism, secondary metabolisms, and amino acid biosynthesis. Two modules, MEgreenyellow (involved in protease, oxidative phosphorylation, endoplasmic reticulum protein processing, folate carbon pool, and glycerol phospholipid metabolism pathways) and MEmidnightblue (involved in protease, endocytosis, steroid biosynthesis, and hippo signaling pathways) were significantly correlated with the strain type. Eight hub genes and two subnetworks were obtained from PPI network. Sub-network A enriched in proteasomes pathway while sub-network B enriched in ribosome pathway. The genes involved in carbon metabolism, secondary metabolic, and amino acid biosynthesis pathways changed significantly under different carbon sources. The changes in proteasome and ribosome activities play roles in carbohydrate metabolism in the methanol-free $\mathrm{P}_{\mathrm{AOX} 1}$ start-up Mut strain.
\end{abstract}

Keywords: Pichia pastoris, RNA-seq, Carbon sources, AOX1 promoter

\section{Introduction}

Pichia pastoris (P. pastoris) is a widely used recombinant expression system for exogenous genes having prokaryotic advantages of having a simple operation and rapid growth, and also has eukaryotic cell functions of posttranslational modifications of proteins. Yeast expression systems include Saccharomyces cerevisiae system,

\footnotetext{
*Correspondence: xszhou@ecust.edu.cn

1 State Key Laboratory of Bioreactor Engineering, East China University of Science and Technology, 130 Meilong Road, Shanghai 200237, China Full list of author information is available at the end of the article
}

methylotrophic yeast expression system, and merozoite yeast expression system (van den Hazel et al. 1996). The methylotrophic yeast $P$. pastoris expression system is one of the most commonly used expression systems.

The most commonly used promoter on P. pastoris expression system is the alcohol oxidase 1 promoter (AOX1), which is a carbon source-dependent promoter whose transcription expression is strongly induced by methanol, but inhibited by other carbon sources, such as glucose, glycerol, or ethanol (Liang et al. 2014). P. pastoris synthesizes a large number of enzymes when using methanol as the sole carbon source. However, the 
use of methanol has many disadvantages as it is toxic, flammable, and volatile, which makes methanol harmful to the human body (Polupanov et al. 2012; Portnoy et al. 2011). Methanol fermentation requires large amounts of oxygen, around three to four times higher than the amount needed when the carbon source is glucose (Lin et al. 2000). It also produces hydrogen peroxide $\left(\mathrm{H}_{2} \mathrm{O}_{2}\right)$ during methanol metabolism, causing oxidative stress and proteolytic degradation of some proteins (Wang et al. 2010).

Researchers have worked on modifying the expression system of $P$. pastoris by various methods which avoid the use of methanol as a carbon source (Lu et al. 2007). The most convenient method to avoid methanol dependence was to engineer other highly expressed promoters that can replace $A O X 1$ and work without methanol induction (Chen et al. 2007). AOX1 promoter has several regulatory factors, such as Mig1, Mig2, Nrg1, Mit1, and Prm1. Among these factors, Mig1, Mig2, and Nrg1 inhibit $A O X 1$ promoter in cells grown in glucose and glycerol, while Mit1 and Prm1 activate AOX1 promoters in cells grown in methanol (Prielhofer et al. 2013). Nevertheless, the regulatory mechanism of $A O X 1$ promoter in $P$. pastoris remains unclear.

An earlier study (Lin-Cereghino et al. 2006) carried out a large-scale mutation screening analysis to identify regulatory factors and to elucidate their mechanisms and the studies also not recorded how to construct an engineered strain or to verify the success of the strain construction. To understand the effects of transcriptional repressors on $A O X 1$ and the transcriptional differences between carbon-derepressed strain (Mut) and wild-type (WT) strain, we modified expression levels of carbon source repressors in P. pastoris (Wang et al. 2017). Mig1, Mig2, and Nrg1 which act as carbon source repressors were inhibited and Mit1 levels were enhanced in the carbon-derepressed strain (Wang et al. 2017). In the present study, we analyzed the transcriptomes of WT and the carbonsource derepressed strain $\Delta m i g 1 \Delta m i g 2 \Delta n r g 1$-Mit1 (Mut) of $P$. pastoris under different carbon sources using RNAseq method. Differential expression genes (DEGs) analysis was applied on different carbon sources with Mut and WT strains. Weighted gene co-expression network analysis (WGCNA) and Kyoto encyclopedia of genes and genomes (KEGG) enrichment were used to explore the effect of different carbon sources on the efficiency of gene expression. Protein-protein interaction (PPI) network analysis was used to obtain key sub-networks and hub genes with Mut and WT strains. The goal of the study was to investigate the mechanisms underlying the carbon derepression and $A O X 1$ promoter regulation

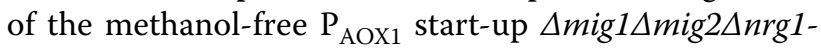
Mit1 strain.

\section{Materials and methods}

Pichia pastoris strains and culture conditions

Pichia pastoris wild-type (WT) strain GS115 (Life Technologies, Carlsbad, CA, USA) and the genetically engineered P. pastoris strain $\Delta m i g 1 \Delta m i g 2 \Delta n r g 1-M i t 1$ (Mut) (constructed and preserved in our laboratory) were used for this study. A chemostatic cultivation medium for recombinant $P$. pastoris was used to provide a stable environment to ensure the reliability and repeatability of RNA-seq analysis. In brief, Mut and WT strains (stored at $-80{ }^{\circ} \mathrm{C}$ ) were inoculated directly into $250-\mathrm{mL}$ shaking flasks containing $50 \mathrm{~mL}$ yeast nitrogen-base/dextrose (YND) medium $(0.67 \%$ yeast nitrogen base (YNB) and $1 \%$ glucose) or into $50 \mathrm{~mL}$ minimal glycerol media (MGY) as the primary seed culture till $\mathrm{OD}_{600}=2-6$, respectively, and cultivated at $30{ }^{\circ} \mathrm{C}$ for $24 \mathrm{~h}$ with agitation (200 rpm). Then, Mut and WT seed cultures were used to inoculate into $500 \mathrm{~mL}$ YND or MGY medium $(0.67 \%$ YNB + 1\% glycerin) till $\mathrm{OD}_{600}=2-6$, respectively, as the second seed cultures and the cultures were then cultivated for $16 \mathrm{~h}$ at $30{ }^{\circ} \mathrm{C}(200 \mathrm{rpm})$. Batch cultivated was started by inoculating the secondary seeds at a concentration of 1:10 (v/v) into a fermenter containing basal salts media (BSM). Batch fermentations were carried out at $30{ }^{\circ} \mathrm{C}$, $\mathrm{pH} 5.0$ (controlled by $25 \%$ ammonium hydroxide), and a minimum of $30 \%$ dissolved oxygen concentration. When carbon source in the medium was depleted and the concentration of dissolved oxygen increased sharply, the chemostat medium using glucose $\left(20.0 \mathrm{~g} \mathrm{~L}^{-1}\right)$, glycerol $\left(20.0 \mathrm{~g} \mathrm{~L}^{-1}\right)$, or methanol $\left(20.0 \mathrm{~g} \mathrm{~L}^{-1}\right)$ as the sole carbon source was supplemented. The fermentation broth is drained by a peristaltic pump to maintain a constant volume of the fermentation broth and the flowrate of glucose, glycerol, or methanol. Finally, they were inoculated into a $50 \mathrm{~mL}$ liquid MGY medium with an initial concentration of $\mathrm{OD}_{600}=1$, and two parallel samples were inoculated for each strain. Samples were taken when the specific growth rate reached $\mu=0.03 \mathrm{~h}^{-1}$.

\section{RNA extraction, construction of cRNA Library and High-throughput sequencing}

The detection of RNA samples was provided by Beijing Novosource Bioinformation Technology Co. RNA concentration was measured using Qubit ${ }^{\circledR}$ RNA Assay Kit with Qubit ${ }^{\circledR}$ 2.0 Fluorometer (Life Technologies, CA, USA), and RNA integrity was assessed with the RNA Nano 6000 Assay Kit by the Agilent 2100 Bioanalyzer (Agilent Technologies, USA), and only the qualified samples were used for library construction and sequencing experiments. As shown in Table 1, a total of 12 samples were used for RNA-seq analysis, including WT strains in glucose, glycerol, and methanol conditions (named as W_D, W_G, and W_M) and Mut strain in the same three 
Table 1 The $P$. pastoris samples used for sequencing

\begin{tabular}{llll}
\hline \multicolumn{3}{c}{ Carbon sources } & \\
\cline { 2 - 4 } & Dextrose (D) & Glycerol (G) & Methanol (M) \\
\hline $\begin{array}{c}\text { Strain type } \\
\text { Mut (M) } \\
\text { Duplicate 1 }\end{array}$ & M_D_1 & M_G_1 & M_M_1 \\
Duplicate 2 & M_D_2 & M_G_2 & M_M_2 \\
Wild-type (W) & & & \\
Duplicate 1 & W_D_1 & W_G_1 & W_M_1 \\
Duplicate 2 & W_D_2 & W_G_2 & W_M_2 \\
\hline
\end{tabular}

Mut indicates the genetically engineered $P$. pastoris strain $\Delta$ mig $1 \Delta m i g 2 \Delta n r g 1$ Mit1

conditions (named as M_D, M_G, and M_M). Each sample was done in duplicates. Illumina Hiseq2000 (Illumina, NY, USA) high-throughput sequencing system was used to sequence RNA of the samples.

\section{Data pre-processing}

Quality control of raw reads was processed by removing reads containing adapter sequences and reads with $\mathrm{N}$ (information cannot be determined) bases greater than $10 \%$. Consequently, clean reads were obtained for downstream analysis. Alignment of the paired-end clean reads to the coding sequence (CDS) of GS115 in The European Bioinformatics Institute (EMBL) database was performed using TopHat v2 (Kim et al. 2013). Based on the predicted gene model of Cufflinks (Trapnell et al. 2012), the alternative splicing event on each sample was classified and counted by Asprofile software (Florea et al. 2013). All the data obtained from reads were assembled together with Cufflinks and then compared with known gene models by Cuffcompare software to predict new transcripts. Gene expression levels were quantified as the number of reads per kilobase of exon region per million reads (RPKM).

\section{DEG analysis}

DEGs were analyzed using the $R$ package DEseq (Anders and Huber 2010; Anders and Huber 2012). For the samples with biological repetition, the original count was corrected by DEseq and the log of the count ratio between the two groups was calculated. Finally, $\log _{2}$ FoldChange (FC) value was obtained. Benjamini \& Hochberg's method (Benjamini and Hochberg 1995) was used for multiple testing correction and genes with an adjusted $P$-value $<0.05$ was selected as the cut-off criterion for the identification of DEGs.

DEGs were analyzed for growth in different sources, including W_G vs. W_D, M_G vs. M_D, W_M vs. W_D, M_M vs. M_D, W_M vs. W_G, and M_M vs. M_G and a total of 6 groups of DEGs were analyzed. Then, we analyzed the DEGs intersection between Mut and WT strains in the same carbon comparison, such as the intersection DEGs between W_G vs. W_D and M_G vs. M_D. Then, a total of three groups of intersection DEGs were determined. Finally, we combined these three groups of intersection DEGs together, which were thought to be genes related to carbon sources.

\section{WGCNA network construction}

Using weighted gene co-expression analysis (WGCNA) $R$ package (Langfelder and Horvath 2008), a co-expressed network of DEGs related to their carbon sources was constructed, and further screening of the gene sets which were significantly related to their strain type, and genes which were considered to be both related to the carbon source and the strain type were selected. Two methods were used to find modules related to the strain type. First, we calculated the correlation between each module feature vector gene and strain types. Second, the mean value of the correlation between the trait and gene expression in each module was taken as the significance. The greater the significance, the more the correlation between the module and the trait. By selecting the weight value of power, the whole network was distributed as a scale-free network, and the power with structure in $R^{2}$ reached 0.8 would be generally selected. Because of batch-related effects, sample heterogeneity, and experimental conditions used, we selected power $=9$ and then, based on clustering and dynamic pruning of the data (Barabási 2009), we clustered the highly correlated genes into modules. Finally, gene sets were identified significantly related to the type of strain.

\section{Functional enrichment analysis}

KOBAS 3.0 (http://kobas.cbi.pku.edu.cn/anno_iden. php) (Xie 2011; Wu et al. 2006) software was used to analyze the Kyoto Encyclopedia of Genes and Genomes (KEGG) database (Kanehisa and Goto 2000) with DEGs related to carbon sources. Then, we also analyzed the KEGG database for genes obtained from the modules of WGCNA analysis with a cut-off of $P$ value $<0.05$ and gene count $\geq 2$, and these pathways were significantly related to the strain type and carbon source.

\section{Construction of protein to protein (PPI) networks}

Search tool for the retrieval of interacting genes (STRING v 10.0) (Szklarczyk 2014) was used to explore interactions between proteins. Komagataella pastoris was selected as the reference species to predict whether there were interactions between matched protein sets. Parameter PPI score was set to 0.7 (high confidence), which requires the interacting protein nodes to be included in the above module genes. Then, the PPI network was 
built by Cytoscape software (version 3.2.0) (Shannon 2003) and cytoNCA plug-in (Tang et al. 2015) (v.2.1.6, http://apps.cytoscape.org/apps/cytonca) was used to analyze the topological properties of the node network. The results included degree centrality (DC), Bweenness Centrality $(\mathrm{BC})$, and closeness centrality $(\mathrm{CC})$, and the hub proteins were obtained by ranking the network topological properties of each node. We used MCODE (Degree Cutoff $=2$, Node Score Cutoff $=0.2, \mathrm{~K}$-core $=2$, MaxDepth $=100$ ) (Bandettini et al. 2012) in Cytoscape to analyze the subnetworks in the PPI network. The modules with score $>5$ were chosen to perform the KEGG pathway analysis using KOBAS 3.0.

\section{Results}

\section{DEGs analysis}

After data pre-processing, a total of 5381 genes in the gene matrix were obtained from 12 samples. According to the cut-off criterion used, a pairwise comparison was used to compare the three different carbon sources (Fig. 1a). There were 399 overlapping DEGs between W_G vs. W_D and M_G vs. M_D. There were 1863 overlapping DEGs between W_M vs. W_D and M_M vs. M_D. In addition, there were 2098 overlapping DEGs between W_M vs. W_G, and M_M vs. M_G. By combining the union DEGs of the Mut strain and WT strain grown under different carbon sources, 2536 DEGs were detected as genes related to carbon sources. Bidirectional hierarchical clustering was carried out to analyze these 2536 DEGs (Fig. 1b). The results of bidirectional hierarchical clustering showed that DEG analysis only separated methanol samples from the three carbon sources, while glucose and glycerol samples were aggregated in the other branches. However, DEGs in glucose and glycerol samples were separated according to the Mut or WT types. Thus, we inferred that these genes were not only associated with carbon source treatment, but also potentially related to the strain type, laying the foundation for our next coexpression analysis.
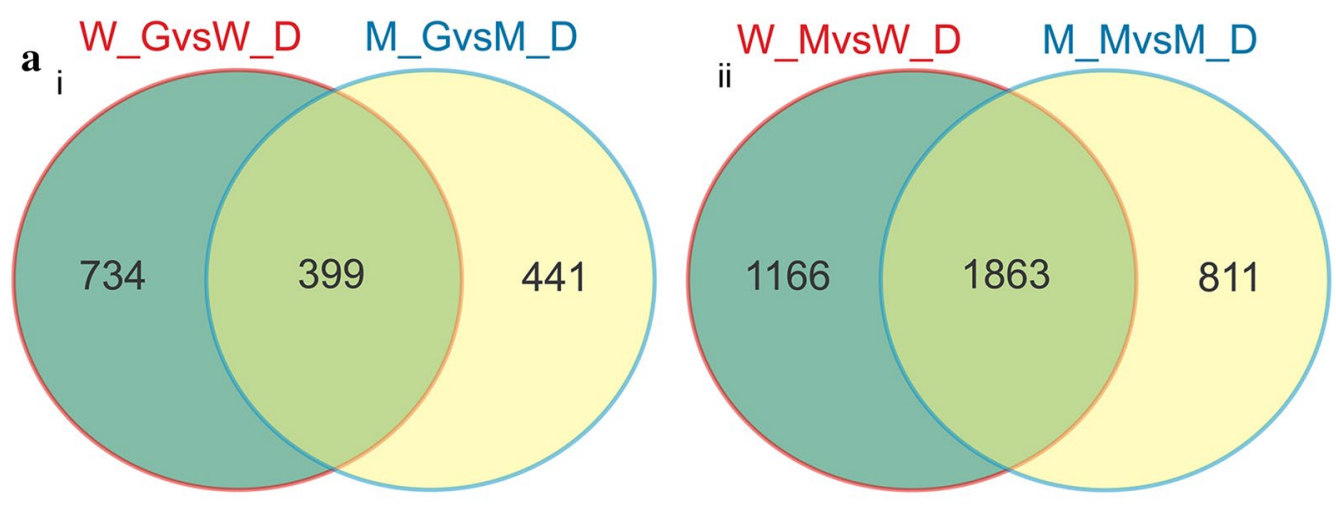

b
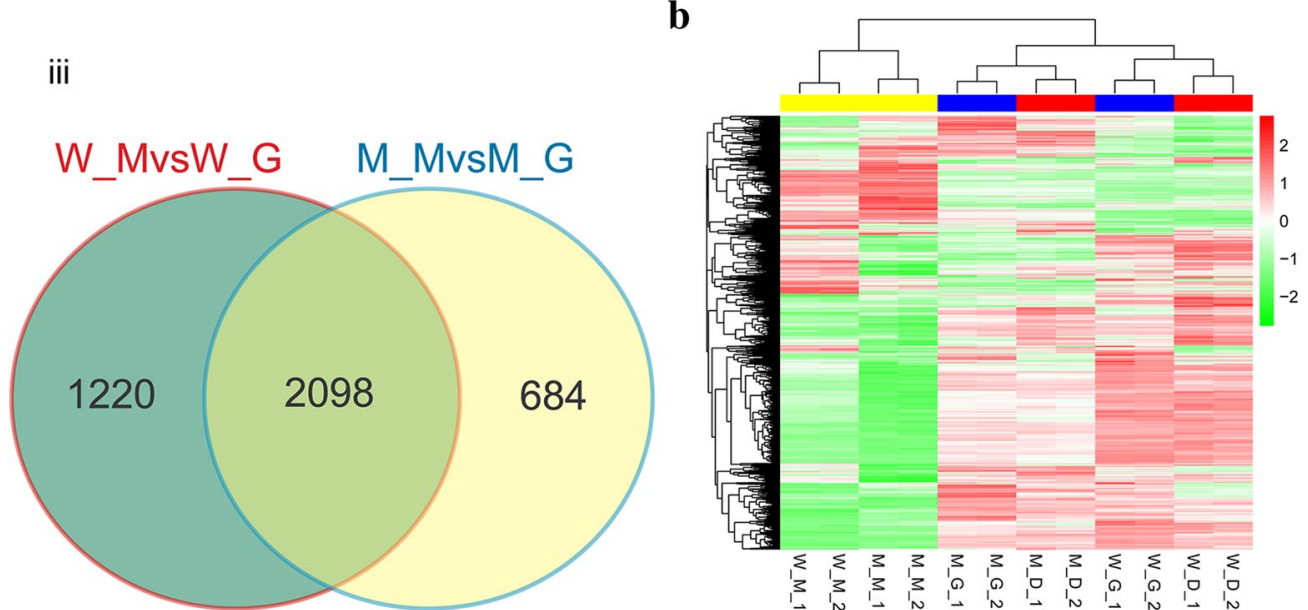

Fig. 1 Statistics and heatmap of DEGs using three carbon sources in $\triangle$ mig1 $\triangle$ mig2 $\triangle$ nrg1-Mit1 (Mut) and wild (WT) strains. a DEG statistics under three carbon sources or strain types: (i) DEGs in Mut or WT strains between dextrose and glycerol groups, (ii) DEGs in Mut or WT strains between methanol and dextrose groups, (iii) DEGs in Mut or WT strains between methanol and glycerol groups. $\mathbf{b}$ DEG heatmap using three carbon sources and different strain types. Red stripes for dextrose, blue for glycerin, and yellow for methanol 
The KEGG enrichment analysis results of the 2536 DEGs showed that with the change of carbon source, the genes involved in carbon metabolism, secondary metabolic pathway, and amino acid biosynthesis changed significantly (Table 2).

\section{Screening of related modules and genes by WGCNA}

To further screen genes related to strain type, we performed WGCNA analysis for the 2536 DEGs (Fig. 2). Based on the clustering and dynamic pruning method, 2536 DEGs were divided into 20 modules (the grey module was a collection of genes that cannot be aggregated into other modules) (Fig. 2a). Modules with heterogeneity coefficient $<0.2$ were merged together, and finally 10 modules were obtained (Fig. 2b, c). By calculating the correlation coefficient between the module eigengene (ME) and sample traits, 4 modules (green-yellow: 122 genes, midnight-blue: 266 genes, grey60: 44 genes, light green: 41 genes) were significantly correlated with the strain type $(p<0.05)$ (Fig. 3a). Both MEgreenyellow and MEmidnightblue modules were significantly correlated with the type of strain (correlation coefficient $>0.9$ ). Genes in the MEmidnightblue module were down-regulated in Mut vs. WT, and genes in the MEgreenyellow module were up-regulated in Mut vs. WT (Fig. 3c-d).

\section{Functional enrichment pathway analysis}

The genes in the MEgreenyellow and MEmidnightblue modules were analyzed by KEGG pathway enrichment, and the results are shown in Fig. 3b. The genes in the MEgreenyellow module were mainly enriched in proteases, oxidative phosphorylation, endoplasmic reticulum protein processing, folate carbon pool, and glycerol phospholipid metabolism pathway, while the genes in the MEmidnightblue module were enriched in proteases, endocytosis, steroid biosynthesis, and hippo signaling pathway (Fig. 3b).

In the carbon methbolism network, the expression level of DAS1, TAL1, PKI1, and MOX were higher in M_G and M_D than in W_G and W_D. The expression level of DAS1, TAL1, PKI1, MOX, FLD1, FHD1, DAK, FBP1 in M_M were higher than in M_G and M_D. Meanwhile,
TKT, ICL1, IDP2, and POX1 expression level in M_M was lower than that in M_G and M_D (Fig. 4).

\section{Construction of PPI networks}

We performed PPI analysis of 388 DEGs in MEgreenyellow and MEmidnightblue modules, and a total of 502 PPI edges and 155 proteins were obtained, as shown in Fig. 5a (Additional file 1: Table S1). The topological properties of PPI network were analyzed. Eight proteins (XP_002489693.1, XP_002490020.1, XP_002491845.1, XP_002492676.1, XP_002493471.1, XP_002493717.1, XP_002493779.1, and XP_002494220.1) obtained high scores in $\mathrm{DC}, \mathrm{BC}$, and $\mathrm{CC}$ within the list of top 30 proteins (Table 3 ), and these 8 proteins were deemed as hub proteins. Five sub-networks were identified by the PPI network function, of which 2 (sub-network A and subnetwork B) had a score $>5$ (Fig. 5b, c). Sub-network A contained 25 genes, of which 8 belonged to the MEgreenyellow module, and the other 17 genes belonged to the MEgreenyellow module. All genes in sub-network B belonged to the MEmidnightblue module. KEGG pathway enrichment was applied to sub-network $A$ and subnetwork $\mathrm{B}$, and the results showed that sub-network $\mathrm{A}$ was enriched in the proteasome pathway, while sub-network B was enriched in the ribosome pathway.

\section{Discussion}

The commonly used and tightly regulated promoter used to drive heterologous protein expression in P. pastoris is the $A O X 1$ promoter; the gene expression of $A O X 1$ is repressed in carbon sources (Brierley et al. 1990). A $P$. pastoris mutant strain expressing AOX1-lac2 fusion has been demonstrated to be able to use sorbitol, alanine, trehalose, or mannitol as the sole carbon source (Inan and Meagher 2001). The deletion of the hexose transporter $\mathrm{PpHXT1}$, leading to glucose or fructose-induced expression of the $A O X 1$ gene and pexophagy, is associated with catabolite repression of Aox in P. pastoris (Zhang et al. 2010). We previously described a novel Mut strain of the $A O X 1$ promoter-based $P$. pastoris expression system, $\Delta m i g 1 \Delta m i g 2 \Delta n r g 1-M i t 1$ (Wang et al. 2017). However, the mechanism underlying the catabolite and $A O X 1$ regulation of this expression system should be explored. In

Table 2 Five KEGG pathways related to carbon source in P. pastoris

\begin{tabular}{llc}
\hline Term & Gene number & $\boldsymbol{P}$-value \\
\hline Metabolic pathways & 395 & $3.20 \mathrm{E}-06$ \\
Biosynthesis of secondary metabolites & 179 & $7.32 \mathrm{E}-05$ \\
Biosynthesis of antibiotics & 140 & 0.000329689 \\
Biosynthesis of amino acids & 83 & 0.000106543 \\
Carbon metabolism & 73 & 0.000570221 \\
\hline
\end{tabular}




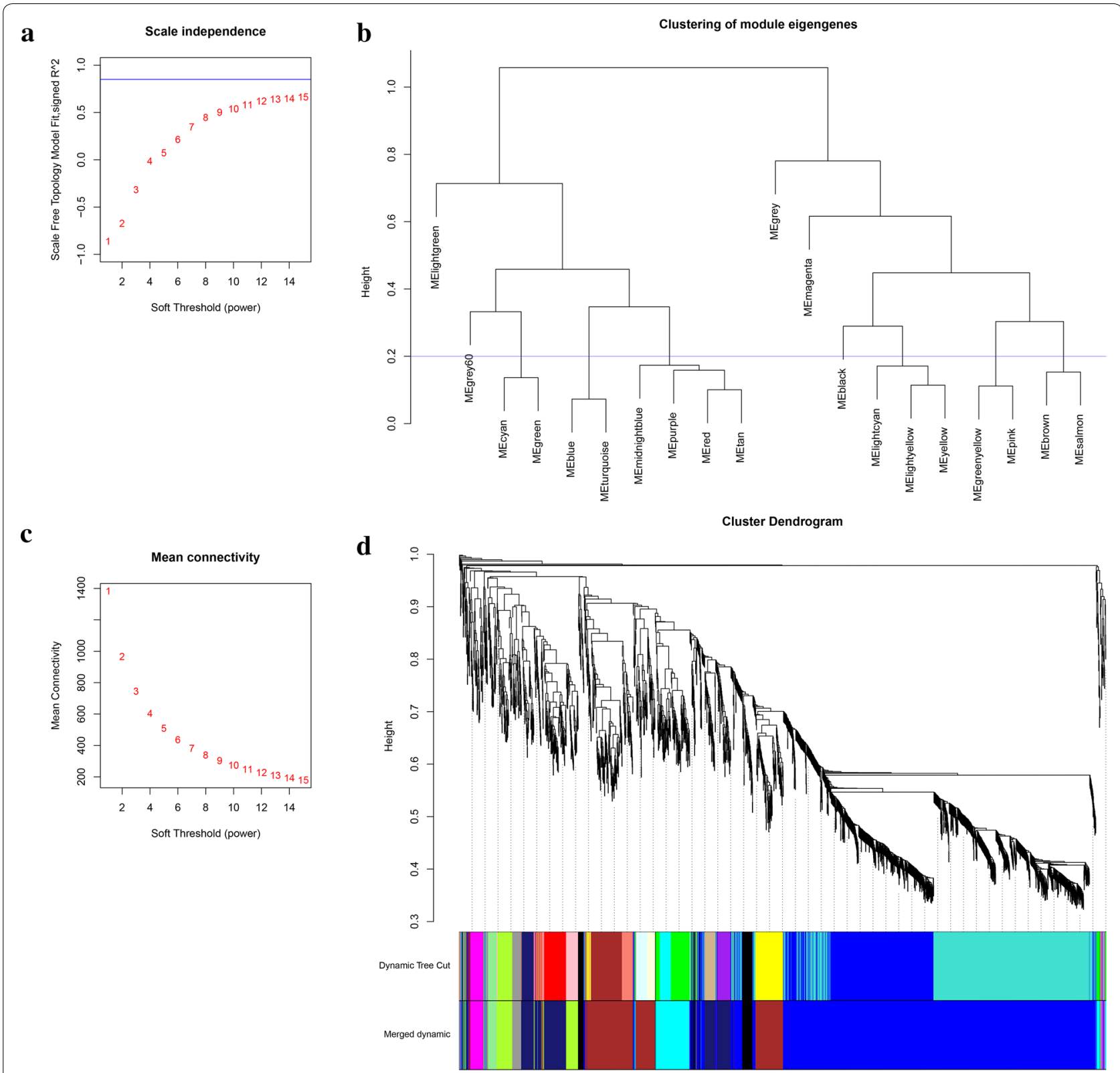

Fig. 2 Power values and module clustering of WGCNA. a Power values of WGCNA, soft threshold (power) representation weight. $\mathbf{b}$ Module clustering of WGCNA, vertical axis denotes dissimilarity coefficient, blue line denotes difference coefficient of 0.2. c The systematic clustering tree of genes and the gene modules generated by dynamic shearing in which different colors represent different gene modules, while the grey part represents genes that cannot be merged into any other module

this study, we compared the gene expression patterns of the Mut strain cultured in three carbon sources (glucose, glycerol, and methanol). A total of 2536 carbon sourceassociated DEGs were detected from the intersection of three groups, and 388 DEGs were found to be associated with both the carbon source type and strain type (Mut or WT). Further investigation of these DEGs indicated the significant involvement of the proteasome and ribosome pathways. Moreover, several hub genes related to $A O X 1$ regulation and carbon sources were identified.

When the two P. pastoris strains (WT and Mut) were induced by different carbon sources, the DEGs were mainly associated with carbon metabolism, secondary metabolic biosynthesis, and amino acid biosynthesis. Carbon metabolism in microbial cells mainly involves glycolysis, the pentose phosphate pathway, and the three 


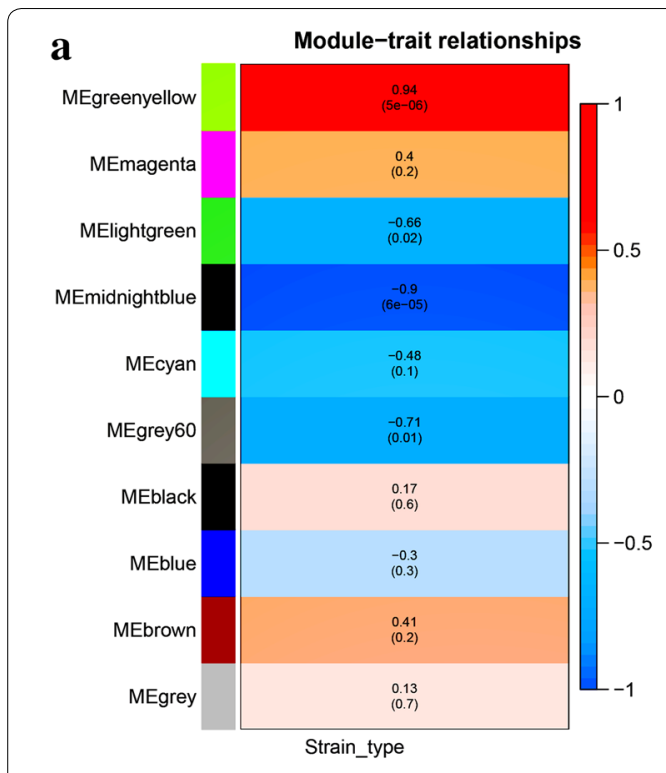

c
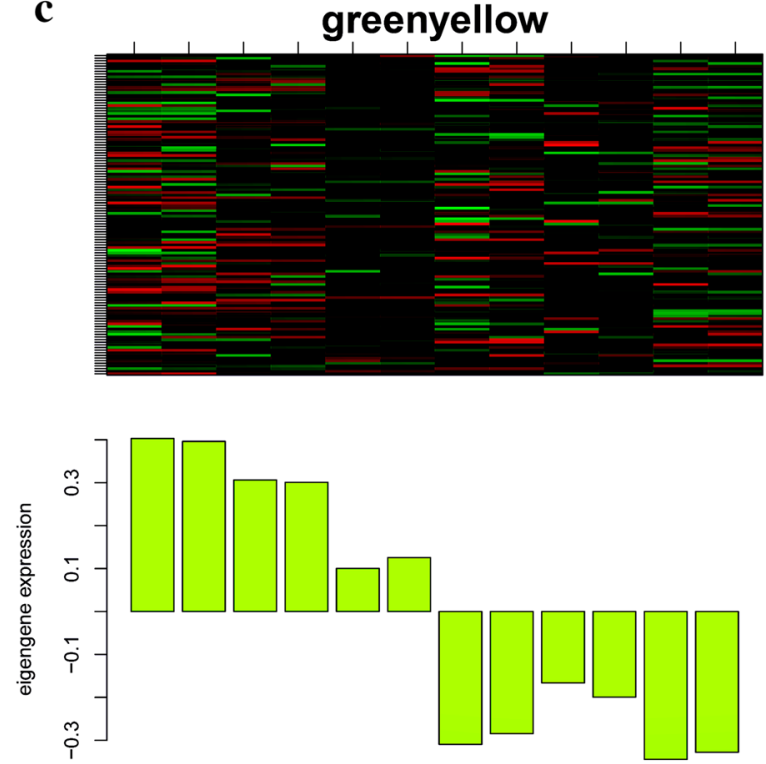

b

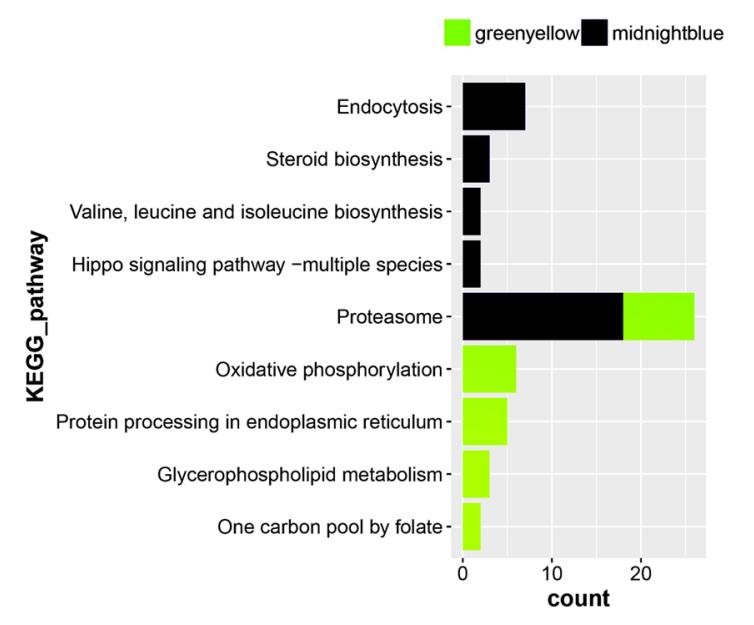

d midnightblue
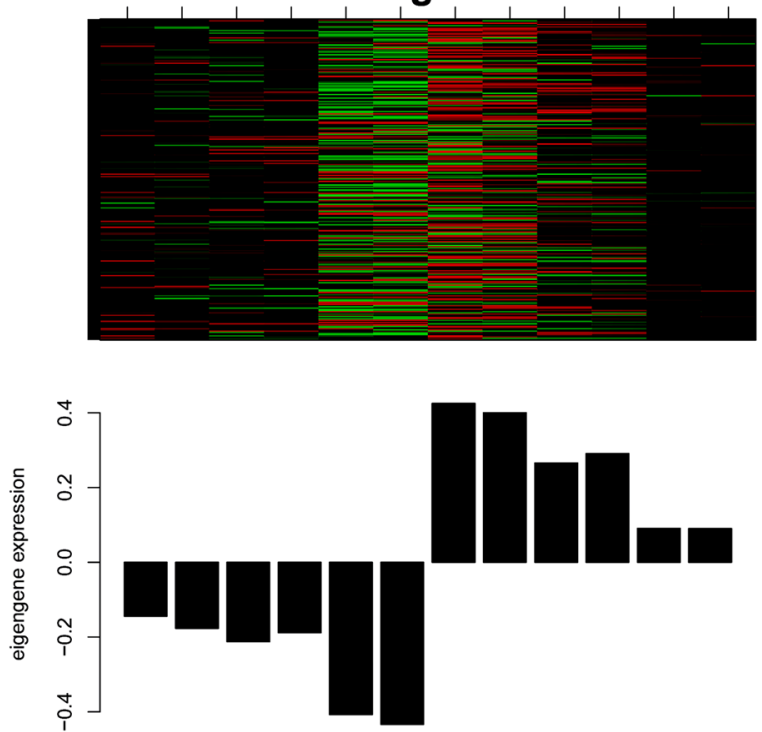

Fig. 3 Correlation analysis of traits in the WGCNA module and their heatmap. a $P$ value and module-trait relationships of each WGCNA modules. b KEGG pathway enrichment of the green-yellow module and midnight-blue module. $\mathbf{c}$, $\mathbf{d}$ Eigen gene expression patterns of the green-yellow module and midnight-blue module: (i) Gene expression heat map; (ii) Eigen gene expression histogram of the modules. Each column of the heat map represents a sample and corresponds to a sample of the gene expression histogram. Each row of the heat map represents a gene

carboxylic acid (TCA) cycle (Zhang et al. 2012). Both the TCA and glyoxylic acid cycles provide energy and precursors for microorganisms (Trapnell et al. 2014). Wang and Yan (2017) showed that when P. pastoris was cultured with glycerol and methanol. Genes associated with the first stage of glycolysis were expressed at the basal level. Dihydroxy acetone phosphate and dihydroxy acetone phosphate were the methanol metabolites in the peroxisome. These metabolites were then transported through the peroxisomal membrane to the cytoplasm and entered the second stage of glycolysis. In a previous study, the growth status of yeast with glucose and methanol as carbon sources was investigated. The metabolic flow of TCA was found to have decreased by three times under methanol growth conditions, while no significant change in transcription or protein levels in the TCA cycle was observed (Russmayer et al. 2015). These results indicate that the TCA cycle may supply different precursor 

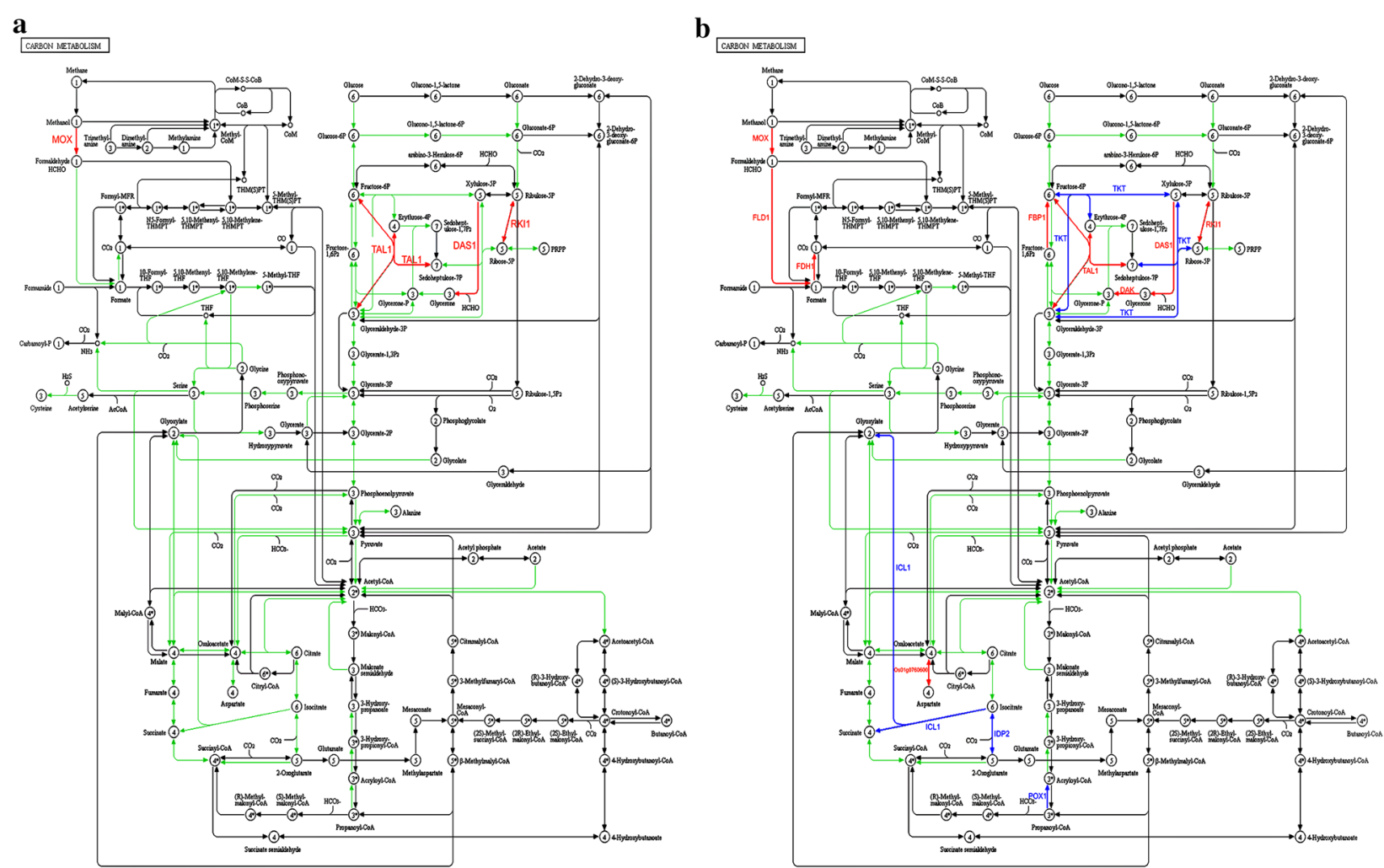

Fig. 4 Carbon metabolism of M_D vs.W_D, M_G vs. W_D (a) and M_M vs. M_D, M_M vs. M_G (b) in P. pastoris. Red words represented the up-regulated genes, and blue words the down-regulated genes

substances for other metabolic pathways when cells are grown under different carbon sources.

The glycerol transporter GT1 may participate in the process of $\mathrm{P}_{\mathrm{AOX} 1}$ inhibition of $P$. pastoris in a medium containing glycerol, and knockdown of this gene can eliminate the glycerol repression of $\mathrm{P}_{\mathrm{AOX} 1}$ (Zhan et al. 2016). In this study, we found several hub proteins in the PPI network of the 388 genes in two significant modules, including XP_002489693.1 (one of six ATPases of the $19 \mathrm{~S}$ regulatory particle of the $26 \mathrm{~S}$ proteasome involved in degradation), XP_002490020.1 (ubiquitin-protein ligase (E3) that interacts with Rpt4p and Rpt6p), XP_002491845.1 (metalloprotease subunit of the 19S regulatory particle of the $26 \mathrm{~S}$ proteasome lid), XP_002492676.1 (Alpha 4 subunit of the 20S proteasome), XP_002493471.1 (Alpha 1 subunit of the 20S core complex of the 26S proteasome), XP_002493717.1 (one of six ATPases of the 19S regulatory particle of the 26S proteasome), XP_002493779.1 (Adenylate cyclase, required for cAMP production and cAMP-dependent protein kinase signaling), and XP_002494220.1 (Beta 6 subunit of the $20 \mathrm{~S}$ proteasome). These proteins were mainly associated with the proteasome and ribosome; one of these proteins was ubiquitin ligases E3, which was a proteolytic enzyme guiding relative protein degradation in the proteasome. The proteasome, which has many catalytic functions and can selectively degrade proteins in cells, is a polyclonal macromolecule complex widely distributed in organisms (Tomko and Hochstrasser 2011). To remove the proteins that the cell does not need for functioning as soon as possible, eukaryotic cells can increase the expression of the proteasome by upregulated transcription of genes involved in the proteasome. Ubiquitin-proteasome-linked protein degradation of the key gluconeogenic enzyme, fructose-1,6-bisphosphatase, is associated with glucose uptake and carbohydrate metabolism of P. pastoris (Santt et al. 2008). Ribosomes are the synthesis machine of proteins, and they are also an important site for cells to perceive the nutrition level within cells. Studies (Beilharz and Preiss 2004; Prielhofer et al. 2015) reported that the proportion of polyribosomes in total ribosomes $(40 \mathrm{~s}, 60 \mathrm{~s}, 80 \mathrm{~s} /$ monosomes and polysomes) increased by approximately twofold, as detected by RNA-seq sequencing, when the different carbon sources induced the expression of proteins in P. pastoris. When methanol was used as the carbon source, high levels of protein expression were observed. At this time, a high number of amino acids need to be 


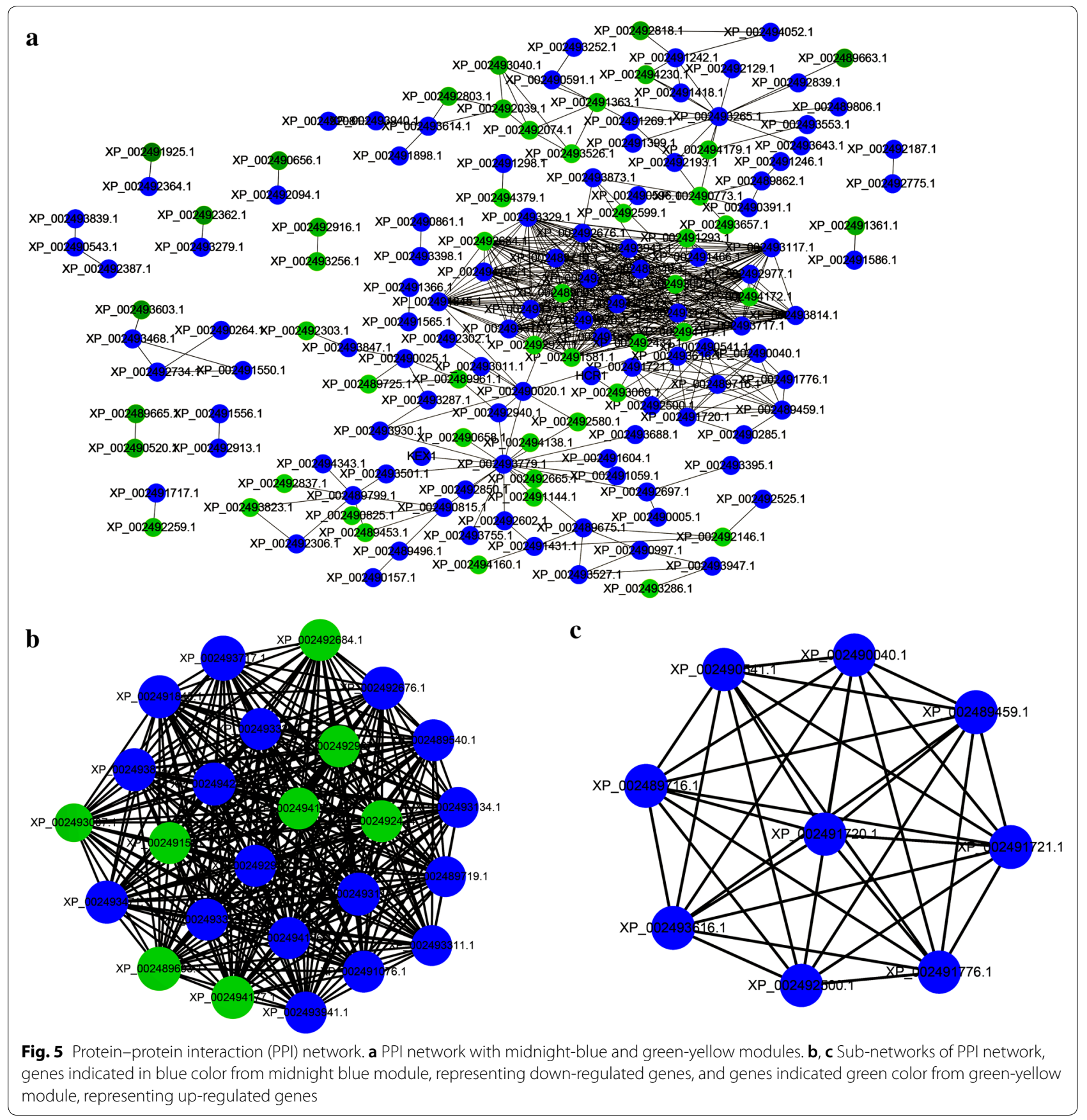

synthesized and more ribosomes need to participate in protein synthesis (Prielhofer et al. 2015). Therefore, we suggested that the changes in ribosomal and proteasomal activities were associated with carbohydrate metabolism and glucose-mediated as well as glycerol-mediated induction of $\mathrm{P}_{\mathrm{AOX} 1}$ in P. pastoris, indicating that the carbon derepressed expression system actually causes protein degradation and amino acid synthesis to meet the demand for heterologous protein secretion. However, the signal transduction mechanism that induces the derepression of $A O X 1$ in $\Delta m i g 1 \Delta m i g 2 \Delta n r g 1-$ Mit1 remains to be investigated further.

In the carbon metabolism network, several genes were up-regulated in M_G and M_D compared with W_G and W_D, such as DASL and FLD1. DAS1 encodes dihydroxyacetone synthase Das and $F L D 1$ encodes formaldehyde dehydrogenase Fld. They are all critical enzymes in the methanol utilization pathway. Both $\mathrm{P}_{D A S 1}$ and $\mathrm{P}_{F L D 1}$ 
Table 3 Top 30 genes with higher topological properties in the protein-protein interaction network

\begin{tabular}{|c|c|c|c|c|c|}
\hline Gene & Degree & Gene & Betweenness & Gene & Closeness \\
\hline XP_002493717.1 & 28 & XP_002490020.1 & 6223.7 & XP_002493717.1 & 0.02147 \\
\hline XP_002489693.1 & 27 & XP_002493779.1 & 6097.7 & XP_002490020.1 & 0.02142 \\
\hline XP_002491845.1 & 26 & XP_002490773.1 & 5526.0 & XP_002489693.1 & 0.02136 \\
\hline XP_002493471.1 & 26 & XP_002493873.1 & 5250.0 & XP_002493873.1 & 0.02136 \\
\hline XP_002492676.1 & 26 & XP_002493717.1 & 4834.5 & XP_002491845.1 & 0.02136 \\
\hline XP_002494220.1 & 26 & XP_002493265.1 & 4645.0 & XP_002493471.1 & 0.02132 \\
\hline XP_002494177.1 & 26 & XP_002491363.1 & 1818.0 & XP_002492676.1 & 0.02132 \\
\hline XP_002493117.1 & 26 & XP_002489675.1 & 1454.0 & XP_002493779.1 & 0.02131 \\
\hline XP_002493814.1 & 26 & XP_002490815.1 & 1346.0 & XP_002490773.1 & 0.02124 \\
\hline XP_002491581.1 & 25 & XP_002492039.1 & 1050.0 & XP_002494220.1 & 0.02122 \\
\hline XP_002493329.1 & 25 & XP_002489799.1 & 864.0 & XP_002494177.1 & 0.02122 \\
\hline XP_002492434.1 & 25 & XP_002489693.1 & 802.5 & XP_002493117.1 & 0.02122 \\
\hline XP_002489540.1 & 25 & XP_002489961.1 & 763.7 & XP_002493814.1 & 0.02122 \\
\hline XP_002492684.1 & 25 & XP_002491845.1 & 753.8 & XP_002493930.1 & 0.02122 \\
\hline XP_002494172.1 & 25 & XP_002493614.1 & 646.0 & XP_002492940.1 & 0.02122 \\
\hline XP_002493375.1 & 25 & XP_002492074.1 & 618.0 & XP_002491581.1 & 0.02122 \\
\hline XP_002494106.1 & 25 & XP_002493040.1 & 618.0 & XP_002493329.1 & 0.02122 \\
\hline XP_002491076.1 & 25 & XP_002493471.1 & 577.6 & XP_002492434.1 & 0.02122 \\
\hline XP_002492927.1 & 25 & XP_002492676.1 & 577.6 & XP_002489540.1 & 0.02122 \\
\hline XP_002492977.1 & 25 & XP_002493501.1 & 500.0 & XP_002492684.1 & 0.02122 \\
\hline XP_002493941.1 & 25 & XP_002490025.1 & 437.7 & XP_002494172.1 & 0.02122 \\
\hline XP_002493311.1 & 24 & XP_002492697.1 & 434.0 & XP_002493375.1 & 0.02122 \\
\hline XP_002489719.1 & 24 & XP_002491242.1 & 432.0 & XP_002494106.1 & 0.02122 \\
\hline XP_002493134.1 & 24 & XP_002491269.1 & 432.0 & XP_002491076.1 & 0.02122 \\
\hline XP_002493007.1 & 22 & XP_002493930.1 & 277.3 & XP_002492927.1 & 0.02122 \\
\hline XP_002493779.1 & 19 & XP_002492193.1 & 249.0 & XP_002492977.1 & 0.02122 \\
\hline XP_002491334.1 & 15 & XP_002491431.1 & 227.0 & XP_002493941.1 & 0.02122 \\
\hline XP_002491293.1 & 15 & XP_002494220.1 & 219.6 & XP_002493311.1 & 0.02122 \\
\hline XP_002493265.1 & 15 & XP_002489862.1 & 218.0 & XP_002489719.1 & 0.02122 \\
\hline XP_002490020.1 & 10 & XP_002492146.1 & 218.0 & XP_002493134.1 & 0.02122 \\
\hline
\end{tabular}

are regulated by methanol (Vogl and Glieder 2013). In the present study, the higher expression level of DASL and FLD1 may play a critical role in carbon metabolism of the dextrose and glycerol medium. TKT plays an important role in the pentose phosphate acid cycle and the photosynthetic reduced pentose phosphate cycle. In the group M_M, the expression level of TKT was down-regulated compared with the group M_G and M_D. This result may indicate that in the case of methanol as the sole carbon source, biotransformation efficiency of the carbon source may be lower in M_M than M_G and M_D. Further results require experimentation to verify.

\section{Conclusion}

In conclusion, with the change of the carbon source, the genes involved in the carbon metabolism pathway, secondary metabolic pathway, and amino acid biosynthesis pathway changed significantly in $P$. pastoris. Changes in the proteasome and ribosome activities played a significant role in the carbohydrate metabolism of the metha-

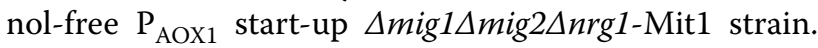
Such an expression system could be an efficient alternative to conventional production methods using methanol as the carbon source.

\section{Supplementary information}

Supplementary information accompanies this paper at https://doi. org/10.1186/s40643-020-00306-w.

Additional file 1: Table S1. Proteins in the PPI network.

\section{Abbreviations}

WT:Wildtype; DEGs: Differentially expressed genes; PPI: Protein-protein interaction; WGCNA: Weighted gene co-expression network analysis; KEGG: Kyoto encyclopedia of genes and genomes; CDS: Coding sequence; RPKM: Reads per kilo bases per million reads; FC: Fold-change; DC: Degree centrality; BC: Bweenness centrality; CC: Closeness centrality; TCA: Three carboxylic acid. 


\section{Acknowledgements \\ Not applicable.}

\section{Authors' contributions}

LS and MC contributed to the conception and design of the research; JW, $X W$, and $Y Z$ acquired data; $X Z$ and $Z S$ performed all the statistical analysis; LS drafted the manuscript; and MC revised the manuscript for important intellectual content. All authors read and approved the final manuscript.

\section{Funding}

None.

\section{Availability of data and materials}

All datasets used and analyzed in the current study are available from the corresponding author on reasonable request.

\section{Ethics approval and consent to participate}

Not applicable.

\section{Consent for publication}

All authors agree to publish this manuscript.

\section{Competing interests}

The authors declare that they have no competing interests.

\section{Author details}

${ }^{1}$ State Key Laboratory of Bioreactor Engineering, East China University of Science and Technology, 130 Meilong Road, Shanghai 200237, China. ${ }^{2}$ Shanghai Collaborative Innovation Center for Biomanufacturing, Shanghai 200237, China. ${ }^{3}$ Bioprocessing Technology Institute, Singapore, Singapore. ${ }^{4}$ Department of Biochemistry, Yong Loo Lin School of Medicine, National University of Singapore, Singapore, Singapore.

Received: 15 November 2019 Accepted: 16 March 2020

Published online: 25 March 2020

\section{References}

Anders S, Huber W (2010) Differential expression analysis for sequence count data. Genome Biol

Anders S, Huber W (2012) Differential expression of RNA-Seq data at the gene level-the DESeq package. Genome Biol

Bandettini WP et al (2012) MultiContrast Delayed Enhancement (MCODE) improves detection of subendocardial myocardial infarction by late gadolinium enhancement cardiovascular magnetic resonance: a clinical validation study. J Cardiovasc Magn Reson 14:83

Barabási AL (2009) Scale-free networks: a decade and beyond. Science 325(5939):412-413

Beilharz TH, Preiss T (2004) Translational profiling: the genome-wide measure of the nascent proteome. Brief Funct Genomic Proteomic 3(2):103-111

Benjamini Y, Hochberg Y (1995) Controlling the false discovery rate: a practical and powerful approach to multiple testing. J R Stat Soc Ser 57(1):289-300

Brierley RA, Bussineau C, Kosson R, Melton A, Siegel RS (1990) Fermentation development of recombinant Pichia pastoris expressing the heterologous gene: bovine lysozyme. Ann NY Acad Sci 589:350-362

Chen HX, Chu J, Zhang SL et al (2007) Intracellular expression of Vitreoscilla hemoglobin improves S-adenosylmethionine production in a recombinant Pichia pastoris. Appl Microbiol Biotechnol 74(6):1205-1212

Florea L, Song L, Salzberg SL (2013) Thousands of exon skipping events differentiate among splicing patterns in sixteen human tissues. F1000 Res 2:188

Inan M, Meagher MM (2001) Non-repressing carbon sources for alcohol oxidase (AOX1) promoter of Pichia pastoris. J Biosci Bioeng 92(6):585-589

Kanehisa M, Goto S (2000) KEGG: Kyoto encyclopedia of genes and genomes. Nucleic Acids Res 28(1):27-30

Kim D, Pertea G, Trapnell C, Pimentel H, Kelley R, Salzberg SL (2013) TopHat2: accurate alignment of transcriptomes in the presence of insertions, deletions and gene fusions. Genome Biol 14:R36

Langfelder P, Horvath S (2008) WGCNA: an R package for weighted correlation network analysis. BMC Bioinformatics 9(1):559
Liang S, Wang B, Pan L, Ye Y, He M, Han S et al (2014) Comprehensive structural annotation of Pichia pastoris transcriptome and the response to various carbon sources using deep paired-end RNA sequencing. BMC Genomics 9(4):511-525

Lin J, Panigraphy D, Trinh L, Folkman J, Shiloach J (2000) Production process for recombinant human angiostatin in Pichia pastoris. J Ind Microbiol Biotechnol 24(1):31-35

Lin-Cereghino GP, Godfrey L, de la Cruz BJ, Johnson S, Khuongsathiene S, Tolstorukov I et al (2006) Mxr1p, a key regulator of the methanol utilization pathway and peroxisomal genes in Pichia pastoris. Mol Cell Biol 26(3):883-897

Lu P, Vogel C, Wang R, Yao X, Marcotte EM (2007) Absolute protein expression profiling estimates the relative contributions of transcriptional and translational regulation. Nat Biotechnol 25(1):117-124

Polupanov AS, Nazarko VY, Sibirny AA (2012) Gss1 protein of the methylotrophic yeast Pichia pastoris is involved in glucose sensing, pexophagy and catabolite repression. Int J Biochem Cell Biol 44(11):1906-1918

Portnoy T, Margeot A, Linke R, Atanasova L, Fekete E, Sándor E et al (2011) The CRE1 carbon catabolite repressor of the fungus Trichoderma reesei: a master regulator of carbon assimilation. BMC Genomics 12:269

Prielhofer R, Maurer M, Klein J, Wenger J, Kiziak C, Gasser B, Mattanovich D (2013) Induction without methanol: novel regulated promoters enable high-level expression in Pichia pastoris. Microb Cell Fact 12(1):5

Prielhofer R, Cartwright SP, Graf AB, Valli M, Bill RM, Mattanovich D, Gasser B (2015) Pichia pastoris regulates its gene-specific response to different carbon sources at the transcriptional, rather than the translational, level. BMC Genomics 16:167

Russmayer H, Buchetics M, Gruber C, Valli M, Grillitsch K, Modarres G, Guerrasio R, Klavins K, Neubauer S, Drexler H, Steiger M, Troyer C, Al Chalabi A, Krebiehl G, Sonntag D, Zellnig G, Daum G, Graf AB, Altmann F, Koellensperger G, Hann S, Sauer M, Mattanovich D, Gasser B (2015) Systems-level organization of yeast methylotrophic lifestyle. BMC Syst Biol 13:80

Santt O, Pfirrmann T, Braun B, Juretschke J, Kimmig P, Scheel H, Hofmann K, Thumm M, Wolf DH (2008) The yeast GID complex, a novel ubiquitin ligase (E3) involved in the regulation of carbohydrate metabolism. Mol Biol Cell 19(8):3323-3333

Shannon P et al (2003) Cytoscape: a software environment for integrated models of biomolecular interaction networks. Genome Res 13(11):2498-2504

Szklarczyk D, et al (2014) STRING v10: protein-protein interaction networks, integrated over the tree of life. Nucleic Acids Res:1003

Tang Y et al (2015) CytoNCA: a cytoscape plugin for centrality analysis and evaluation of protein interaction networks. BioSystems 127:67-72

Tomko RJ Jr, Hochstrasser M (2011) Order of the proteasomal ATPases and eukaryotic proteasome assembly. Cell Biochem Biophys 60(1-2):13-20

Trapnell C, Roberts A, Goff L, Pertea G, Kim D, Kelley DR et al (2012) Differential gene and transcript expression analysis of RNA-seq experiments with TopHat and Cufflinks. Nat Protoc 7:562-578

Trapnell CR, Roberts A, Goff L, Pertea G, Kim D, Kelley DR, Pimentel H, Salzberg SL, Rinn JL, Pachter L (2014) Differential gene and transcript expression analysis of RNA-seq experiments with TopHat and Cufflinks. Nat Protoc 9(10):2513

van den Hazel HB, Kielland-Brandt MC, Winther JR (1996) Biosynthesis and function of yeast vacuolar proteases: review. Yeast 12:1-16

Vogl T, Glieder A (2013) Regulation of Pichia pastoris promoters and its consequences for protein production. New Biotechnol 30(4):385-404

Wang XY, Yan X (2017) The metabolic difference and mechanism of Komagataella phaffii in glycerol and methanol culture based on transcriptomic analysis. Acta Microbiol Sin:1-17

Wang Z, Wang Y, Zhang D, Li J, Hua Z, Du G, Chen J (2010) Enhancement of cell viability and alkaline polygalacturonate lyase production by sorbitol co-feeding with methanol in Pichia pastoris fermentation. Biores Technol 101(4):1318-1323

Wang J, Wang X, Shi L, Qi F, Zhang P, Zhang Y, Zhou X, Song Z, Cai M (2017) Methanol-independent protein expression by AOX 1 promoter with trans-acting elements engineering and glucose-glycerol-shift induction in Pichia pastoris. Sci Rep 7:41850. https://doi.org/10.1038/srep4 1850 
Wu J, Mao X, Cai T, Luo J, Wei L (2006) KOBAS server: a web-based platform for automated annotation and pathway identification. Nucleic Acids Res $34: 720-724$

Xie CEA (2011) KOBAS 2.0: a web server for annotation and identification of enriched pathways and diseases. Nucleic Acids Res 39:316-322

Zhan C, Wang S, Sun Y, Dai X, Liu X, Harvey L, McNeil B, Yang Y, Bai Z (2016) The Pichia pastoris transmembrane protein $\mathrm{GT} 1$ is a glycerol transporter and relieves the repression of glycerol on AOX1 expression. FEMS Yeast Res. https://doi.org/10.1093/femsyr/fow033

Zhang P, Zhang W, Zhou X, Bai P, Cregg JM, Zhang Y (2010) Catabolite repression of Aox in Pichia pastoris is dependent on hexose transporter PpHxt1 and pexophagy. Appl Environ Microbiol 76(18):6108
Zhang CL, Qin ZJ, Wang GZ, Ji ZB, Wang JM (2012) Transcriptome and RNA-Seq technology. Biotechnol Bull 12:51-56

\section{Publisher's Note}

Springer Nature remains neutral with regard to jurisdictional claims in published maps and institutional affiliations.

\section{Submit your manuscript to a SpringerOpen ${ }^{\odot}$ journal and benefit from:}

- Convenient online submission

- Rigorous peer review

- Open access: articles freely available online

- High visibility within the field

- Retaining the copyright to your article

Submit your next manuscript at $\boldsymbol{\nabla}$ springeropen.com 